\title{
The Effects of Non-Unity Lewis Numbers on Turbulent Premixed Flame Interactions in a Twin V-flame Configuration
}

\author{
T.D. Dunstan ${ }^{1}$, N. Swaminathan*1 , K.N.C. Bray ${ }^{1}$, N.G. Kingsbury ${ }^{1}$ \\ 1 Department of Engineering, Cambridge University, Cambridge, CB2 1PZ, UK.
}

\section{*Corresponding author:}

Department of Engineering, Cambridge University,

Trumpington Street, Cambridge, CB2 1PZ, UK.

E-mail: ns341@cam.ac.uk.

Fax: $+44(0) 1223765311$.

Published in Combustion Science and Technology, 185, 874-897, 2013

(Authors' preprint)

Running Title: Non-Unity Lewis Number Flame Interactions. 


\begin{abstract}
The influence of Lewis number on turbulent premixed flame interactions is investigated using Automatic Feature Extraction (AFE) applied to high-resolution flame simulation data. Premixed turbulent twin V-flames under identical turbulence conditions are simulated at global Lewis numbers of $0.4,0.8,1.0$ and 1.2. Information on the position, frequency and magnitude of the interactions is compared, and the sensitivity of the results to sample interval is discussed. It is found that both the frequency and magnitude of normal type interactions increases with decreasing Lewis number. Counter-normal type interactions become more likely as the Lewis number increases. The variation in both the frequency and the magnitude of the interactions is found to be caused by large-scale changes in flame wrinkling resulting from differences in the thermo-diffusive stability of the flames. During flame interactions thermo-diffusive effects are found to be insignificant due to the separation of time scales.
\end{abstract}

Keywords: flame stretch, flame surface density, data registration, turbulent flame modelling

\title{
1 Introduction
}

The effects of multiple flame interactions on turbulent premixed flames is a subject of relevance to several aspects of premixed flame analysis and the design of premixed combustion devices. The merging of interacting flame elements causes a rapid change in the local rate of heat release, and has been identified as a leading cause of combustion generated noise in laminar (Schuller et al., 2002; Candel et al., 2004; Talei et al., 2012) and turbulent (Balachandran et al., 2005) premixed systems. In appropriate conditions this turbulent flame noise can lead to thermo-acoustic instabilities due to coupling between the rate of heat release and pressure fluctuations. A proper understanding of flame interactions is also necessary for the development of robust 
combustion models that are capable of spanning multiple combustion regimes since in the presence of flame interaction the flame behaves neither as a continuous and unbroken flame front, nor as a perfectly-stirred homogeneous reacting mixture.

A method for identifying and extracting information on flame interactions from a three-dimensional time-resolved turbulent flame simulation was introduced in Dunstan et al. (2012). Here, this technique is applied to the study of flame interactions in non-equidiffusive mixtures. The Lewis number, defined as Le $=\alpha / D$ where $\alpha$ and $D$ are the thermal and mass diffusivities of the deficient reactant, characterises the preferential diffusion of heat over species within the flame. Emerging fuel types such as pure hydrogen and Syngas, which has a large hydrogen content, have Lewis numbers significantly below unity when burnt under lean conditions due to the high mobility of the hydrogen atom. More generally, an understanding of the effects of non-unity Lewis numbers is important for the accurate modelling of multi-component and stratified mixtures where the effective Lewis number may vary locally due to changes in composition and local equivalence ratio.

Premixed flame interactions can be categorised as either normal or counter-normal, where the flame normal is defined as positive in the direction of the fresh gases and represents the direction of propagation of the unperturbed laminar flame. Normal interaction (also referred to as upstream interaction in the literature, see Sohrab et al. (1984)) therefore describes the situation where two flame elements approach one another from the fresh gas side. This process has been investigated theoretically (Kollmann and Chen, 1998) and numerically (Chen and Sohrab, 1995). Chen et al. (1999) employed high resolution 2D simulations with methane-air chemistry to identify several key time scales for the interaction process. Normal interaction is characterised 
by an initial acceleration of the flames as the preheat layers begin to merge, followed, at the point of merger, by a topological change to the flame isosurfaces resulting in the formation of cusps with extreme values of negative curvature. The cusp recovery stage involves a rapid loss of flame surface area as the cusps retract from the point of interaction into the fresh gases.

Counter-normal interactions (also referred to as downstream interactions) have been investigated both experimentally and numerically (Sohrab et al., 1984; Lee and Chung, 1994; Kostiuk et al., 1999; Hawkes and Chen, 2004). Counter-normal interactions are produced either by flame elements being brought together against their normal propagation direction by the surrounding turbulence, or due to counter-normal flame propagation, which is known to occur in thermo-diffusively stable flames in areas of high positive curvature (Gran et al., 1996). In general, counter normal interaction occurs over longer time scales compared to normal interactions due to the lack of initial flame acceleration and the slower cusp recovery following merger.

The effects of Lewis numbers on both laminar and turbulent flames have been widely studied (see Lipatnikov and Chomiak (2005) for a review). The reduction in thermo-diffusive stability associated with lower Lewis numbers leads to an increasingly wrinkled turbulent flame structure and higher turbulent flame speeds (Haworth and Poinsot, 1992; Goix and Shepherd, 1993). Ultra-low Lewis number flames also exhibit thermo-diffusive instabilities which can lead to a significant enhancement of the flame wrinkling beyond that produced by the normal turbulent processes of strain and curvature (Goix and Shepherd, 1993; Bell et al., 2007). The effect of Lewis number on laminar flame interactions has been investigated by Chen and Sohrab (1995) using one-dimensional numerical simulations, however, important multi-dimensional effects such as cusp retraction are not taken into account in this configuration. The 
response of non-unity Lewis number laminar flames to steady and unsteady forcing has also been investigated for flames stabilised in a stagnation flow (Law and Sung, 2000), where it was noted that as the frequency of forcing is increased, the response of both equidiffusive and non-equidiffusive flames is gradually attenuated.

The aims of the current paper are to consider how these changes in the mean and local flame structures relate to the rate of production of flame interactions, and to the role of interactions in flame area change. This is done using high-resolution simulations of lean premixed turbulent flames in a twin V-flame configuration with global Lewis numbers of 0.4, 0.8, 1.0 and 1.2. An Automatic Feature Extraction (AFE) technique, described by Dunstan et al. (2012), is employed for systematically identifying interactions and their effects. Furthermore, we consider the sensitivity of the technique to the choice of sample interval, $\Delta t$, which is a key parameter in correctly identifying the area changes associated with individual interactions.

Details of the simulations, numerical methods and a brief summary of the AFE technique are given in Sections 2 and 3 respectively. Results are presented in Section 4 beginning with a description of the mean velocity fields and the influence of the flame holders on the downstream turbulence in Section 4.1. The types and distributions of the interactions are discussed in Section 4.2. The sensitivity to the sample interval, $\Delta t$, and the effects of interactions on the mean flame brush are discussed in terms of their stretch rate contributions in Section 4.5. Conclusions are given in Section 5.

\section{Simulation Details}

Simulations were carried out using the SENGA2 code, an enhanced version of the SENGA code (Jenkins and Cant, 1999) with accommodation for multiple species, tem- 
perature dependent transport properties and modifications to the boundary conditions to preserve the accuracy of the solution during flame-boundary interactions (Dunstan et al., 2011). Fully compressible conservation equations are solved using $10^{\text {th }}$ order central differencing in the interior and a fourth order Runge-Kutta scheme advances the solution in time.

The twin V-flame configuration is illustrated in Fig. 1 which shows a two-dimensional slice taken from a three-dimensional domain. The computational domain is of size $L_{x}=12.8 \mathrm{~mm}, L_{y}=6.4 \mathrm{~mm}$, and $L_{z}=11.9 \mathrm{~mm}$, where $x$ and $y$ are the streamwise and transverse directions as indicated in Fig. 1, and the $z$-direction is statistically homogeneous. The domain is discretised on a uniform grid of $N_{x}=672, N_{y}=336$, and $N_{z}=624$ computational nodes, ensuring a maximum diagonal grid point separation of $\Delta=\sqrt{\Delta x^{2}+\Delta y^{2}+\Delta z^{2}}<\delta_{c} / 15$, where $\delta_{c}$ is the laminar flame thickness to be defined below. Boundary conditions used are non-reflecting inflow/outflows on the downstream and transverse faces as shown in Fig. 1, and periodic in the homogeneous direction. Details of the boundary conditions including the modifications made to allow the passage of the flame through the boundary are discussed by Dunstan et al. (2011)

Fully-developed homogeneous isotropic turbulence from a pre-computed, nonreacting flow simulation is interpolated onto the inlet plane, marked as turbulent inflow in Fig. 1, with a constant mean velocity $\bar{u}_{i n}=10 \mathrm{~ms}^{-1}$. The domain for the precomputed turbulence has a streamwise dimension of $2 L_{x}$ thereby allowing a maximum useable reacting flow simulation time of $2 L_{x} / \bar{u}_{i n}=2.56 \mathrm{~ms}$. The same initial turbulent field is used for all the reacting flow simulations presented here and has an rms velocity fluctuation of $u_{i n}^{\prime}=4.9 \mathrm{~ms}^{-1}$ and an integral length scale of $l_{0 i n}=0.85 \mathrm{~mm}$. 
At the flame holders, velocity and species mass fractions are imposed through Gaussian weighting functions with an approximate diameter of $0.5 \mathrm{~mm}$. The flame holder centres are located at $x=1.5 \mathrm{~mm}$ from the inflow plane and with a separation of $d_{F H}=2.5 \mathrm{~mm}$ in the transverse direction. In contrast to previous simulations of Dunstan et al. (2012), the flame holder velocity in the current cases is set to zero. This is done to recreate a more realistic no-slip condition that exists for experimental flame holders, however, it should be noted that no attempt is made in these simulations to resolve the boundary layer around the flame holders: the velocity gradients are artificially restricted by both the Gaussian weighting functions and the grid resolution, and so the simulations should not be considered as a 'true' DNS in these regions.

Chemistry is approximated with a single-step irreversible reaction between reactants and products. Transport coefficients are temperature dependent and follow fifthorder polynomial functions in the standard NASA format (McBride et al., 1993). The reactant Lewis numbers, Le $=\alpha / D$, where $\alpha$ and $D$ are the thermal and mass diffusivities of the reactants respectively, are constant and specified as simulation parameters prior to initialisation. Values of the thermo-chemical parameters for the current simulations are given in Table 1, where $s_{L}$ is the unstretched laminar flame speed, $T_{a d}$ is the adiabatic flame temperature, and $T_{\text {in }}$ is the inlet reactant temperature. The laminar flame thickness is given by $\delta_{c}=1 / \max |\nabla c|$, where $c$ is the progress variable which is equivalent to the product mass fraction $c=Y_{p}$ under the single-step assumption. The thermal thickness is given by $\delta_{t h}=\left(T_{a d}-T_{i n}\right) / \max |\nabla T|$, and the diffusive thickness is $\delta=D / s_{L}$. The flame time scales based on the thermal thickness and progress variable are $\tau_{t h}=\delta_{t h} / s_{L}$ and $\tau_{c}=\delta_{c} / s_{L}$ respectively. The value of the heat release parameter $\tau=2.52$ used in this study is typical of lean preheated flames used in gas turbine combustion (Jones, 2011) 
Table 2 summarises the relevant non-dimensional parameters based on the conditions at the inlet for the flames simulated in this study. The Karlovitz number is approximated using $K a \approx\left(u_{i n}^{\prime} / s_{L}\right)^{\frac{3}{2}}\left(l_{0 i n} / \delta\right)^{-\frac{1}{2}}$, and the Damköhler number is Da= $\left(l_{0 i n} s_{L}\right) /\left(u_{i n}^{\prime} \delta_{c}\right)$. These flames are therefore representative of lean preheated premixed hydrocarbon-air flames in the thin reaction zones regime, as defined in the modified combustion regime diagram of Peters (2000). The turbulence Reynolds number at the inflow plane is $R e_{l_{0}}=u_{i n}^{\prime} l_{0 i n} / v_{i n}=82$, where $v_{i n}$ is the kinematic viscosity of the inflowing mixture, for all the flames simulated in this study.

\section{Data Analysis}

After initialising the fields, the simulations are allowed to evolve for a minimum of one flow-through time, $\tau_{F T}=L_{x} / \bar{u}_{i n}$, or until a stationary state has been reached. The criterion used to identify a stationary state is described in Section 4. Mean fields are two dimensional and constructed by time and space averaging over all grid points in the homogeneous direction and all snapshots in time after a stationary state has been reached. For any variable $Q(x, y, z, t)$ :

$$
\bar{Q}(x, y)=\frac{1}{S N_{z}} \sum_{m=1}^{S} \sum_{k=1}^{N_{z}} Q\left(x, y, k, t_{m}\right)
$$

where $S$ is the total number of equally spaced snapshots in time $(S=64,61,53$, and 41 for flames A-C respectively), and $N_{z}$ is the number of grid points in the periodic direction respectively. Density-weighted or Favre averages are obtained through $\widetilde{Q}=\overline{\rho Q} / \bar{\rho}$.

To extract information on the time, position and change in flame area associated 
with individual interactions a technique called Automatic Feature Extraction (AFE) was applied. The details of this technique are explained in Dunstan et al. (2012), and thus only a brief summary will be given here. Automatic Feature Extraction uses data registration based on the Dual-Tree Complex Wavelet Transform (DTCWT), developed by N. Kingsbury (Chen and Kingsbury, 2012; Kingsbury, 2001), in which two related data sets can be aligned according to their shared features. In the current context, this involves taking two snapshots of the progress variable field at successive times $t_{i}$ and $t_{i+1}$ separated by an interval $\Delta t$ and aligning the two snapshots such that differences due to convection, strain and curvature are eliminated. Due to the properties of the applied transformation matrix, however, the topology of each progress variable field is preserved, such that by subtracting the registered snapshots from one another, only areas where a change in the local topology has occurred remain. Since, by definition, changes in topology can only occur as a results of flame interaction, the flame interactions occurring within the interval are systematically revealed. These extracted regions, $\Phi$, can then be conditioned on the original snapshots to establish the interaction flame surface areas, $\phi_{i}$ and $\phi_{i+1}$ associated with the snapshots at times $t_{i}$ and $t_{i+1}$ respectively.

A key consideration in applying this process is in the choice of the sample interval $\Delta t$. This issue is addressed in detail in Section 4.5 in relation to the flame stretch, since this has the most pronounced sensitivity to $\Delta t$ and is an important quantity in turbulent combustion modelling. An upper limit on $\Delta t$ must be set to ensure that not more than one interaction is included in each extracted region. This is achieved in the current simulations if $\Delta t \leq 0.02 \mathrm{~ms}$, which results in a large number $(\approx 128)$ of snapshot pairs requiring analysis. To facilitate this process, we therefore consider binarised progress variable fields $c^{*}$, where $c^{*}=1$ if $c>0.8$ and otherwise $c^{*}=0$, since the $c=0.8$ value is close to the position of maximum reaction rate in the laminar flames for the 
thermo-chemistry considered here. It should be noted that the topological changes to other isosurfaces within the flame will not necessarily follow the same trends as the $c=0.8$ isosurface. However, from a modelling point of view, changes to other parts of the flame matter only to the extent that they affect the rate of heat release within the reaction layer, and for the present analysis this is adequately captured by considering only a single isosurface at $c=0.8$.

\section{Results}

Before data can be collected for analysis, all initial transients must have decayed and a stationary state should have been reached. To determine when this situation occurs the global average mass fraction of products, $\Omega$, is tracked throughout the simulations. The

quantity $\Omega$ is simply the volume average of the progress variable $\Omega=\int_{V} c d v / V$, where $V$ is the computational domain volume. This quantity varies from 0 at initialisation to 1 when the computational volume contains only fully burnt products. Figure 2 shows the temporal variation of $\Omega$ for all the flames, and a clear trend can be seen with increasing time. A stationary state is reached quickly when the Lewis number is low, which reflects the faster turbulent flame speeds associated with lower Lewis number flames (Abdel-Gayed et al., 1984; Lipatnikov and Chomiak, 2005). There is about a $1 \mathrm{~ms}$ difference between the $\mathrm{Le}=0.4$ and $\mathrm{Le}=1.2$ flames as suggested by Fig. 2 . The starting points for data analysis are marked in Fig. 2 by vertical dashed lines. Since the statistics are sufficiently converged in all cases, the reduced number of samples available for the higher Lewis number flames does not affect any of the conclusions reached in this paper. 


\subsection{Mean Flow Fields}

Profiles of the Favre-averaged streamwise velocity, $\tilde{u}$, and rms velocity fluctuations $u^{\prime}$ are shown in Fig. 3. In Fig. 3a, $\tilde{u}$ is plotted along the centre line of the domains at $y=L_{y} / 2$ for all the flames in Table 1 . A large acceleration of the flow in the region of the flame holders around $x=1.5 \mathrm{~mm}$ can be seen for all the flames as a result of the deflection of the streamlines around the flame holders. Since this is an effect of the fluid dynamics the acceleration does not depend on the Lewis number, as one can observe from Fig. 3a. Immediately downstream of the flame holders the differential effects of heat release on the flow become apparent, with a general trend of increased steamwise acceleration with decreasing Lewis number caused by the higher turbulent flame speeds of the lower Lewis number flames. Transverse profiles of $\tilde{u}$ and $u^{\prime}$ are shown respectively in Fig $3 \mathrm{~b}$ and $\mathrm{c}$ for flame $\mathrm{C}$ at streamwise positions $x=1.5,4.0,6.5,9.0$, and $11.5 \mathrm{~mm}$ as indicated in Fig. 1. The profiles for all the flames in the database are qualitatively similar. The profiles at $x=1.5 \mathrm{~mm}$ pass through the centre of the flame holders and demonstrate the steep velocity gradients present in this region. Small areas of recirculation are formed behind the flame holders (not visible in Fig. 3b). These extend a maximum of $1.6 \mathrm{~mm}$ downstream of the flame holder centre (for Flame D), and less for flames A-C because of the expansion of the burnt gases and subsequent acceleration of the flow. The presence of steep velocity gradients around the flame holders suggests that shear generated turbulence might be significant in the downstream region, however, this does not appear to be the case, as illustrated in Fig 3c. Small increases in $u^{\prime}$ can be seen at $x=4 \mathrm{~mm}$ but in general, shear generated turbulence is not significant compared to the more general attenuation of turbulence in the burnt gas region.

It is worthwhile also to consider characteristics of the turbulence experienced by the flames. Although the turbulence at the inlet is approximately homogeneous and 
isotropic, the flame holders impose severe directional constraints on the flow. While this may be a better representation of the effects of real flame holders, it is also important to understand how specific the resulting turbulence is to the V-flame configuration and therefore how far the results presented here may be generalised to other flame configurations.

The quantities of interest in this regard are the two anisotropy invariants $\xi$ and $\eta$ (Pope, 2000). These two quantities fully characterise the normalised Reynolds stress anisotropy tensor $b_{i j}=\left(\overline{u_{i}^{\prime \prime} u_{j}^{\prime \prime}}\right) /\left(\overline{u_{k}^{\prime \prime} u_{k}^{\prime \prime}}\right)-\delta_{i j} / 3$, where $u_{i}^{\prime \prime}=u_{i}-\tilde{u}_{i}$ and $\delta$ is the Kronecker delta function. These invariants can be obatined from the eigenvalues of the anisotropy tensor and are defined as $6 \xi^{3}=b_{i j} b_{j k} b_{k i}$ and $6 \eta^{2}=b_{i j} b_{j i}$. Together, $\xi$ and $\eta$ provide a local measure of the type and degree of anisotropy of the turbulence. They approach zero only in fully isotropic flows, but otherwise take values in the range $-1 / 6<\xi<1 / 3$ and $0<\eta<1 / 3$ (Pope, 2000). Figures 4a and $4 \mathrm{~b}$ show the variation of $\xi$ and $\eta$ for flame B. Similar distributions exist in all the flames. The effect of the flame holders can clearly be seen in the near maximal values of the invariants in these regions and in the shear layers immediately downstream, indicating strongly anisotropic, single-component Reynolds stresses in these areas. The effects persist downstream although a gradual return to isotropy can be seen. Nevertheless, in the central region where the majority of flame interactions take place (to be discussed in the next section), the flow remains approximately isotropic throughout. These observations suggest that interactions occurring in the central region are unlikely to be strongly affected by the anisotropy introduced by the flame holders and so should be representative of interactions occurring under similar turbulence conditions in other flame geometries. However, interactions occurring in the highly anisotropic shear layers immediately downstream of the flame holders, are likely to be more strongly configuration specific and thus due caution must be exercised when generalising their 
behaviour to other types of flame.

\subsection{Type and Position of Interactions}

As noted in the introduction, the flame interactions can be broadly categorised as either normal or counter-normal depending on the direction of approach of the flame elements relative to the locally defined flame normal $\vec{N}=-\nabla c /|\nabla c|$. Three sub-types can also be identified within each of these categories, depending on the precise topological changes that occur during the interaction, to give six interaction types in total. These are illustrated schematically in Fig. 5 for normal-type interactions. Counter-normal interactions are topologically identical but with reactants and products reversed.

The six sub-types are: convex-normal (CX), tunnel-closure (TC), and pocket burnout (PB) for normal sub-types, and counter-normal (CN), counter tunnel-closure (CTC), and counter pocket burn-out (CPB) for the counter-normal sub types. In contrast to the previous results of Dunstan et al. (2012), two additional sub-types have been identified in the current data: CTC and CPB, both of which occur only for Le $>1$ in flame D. It was argued in Dunstan et al. (2012) that the CTC and CPB type interactions, although theoretically possible, were unlikely to occur in practice due to the normal flame propagation direction and strain rate relations of the surrounding fluid. The assumption of normal flame propagation, however, is incorrect for thermo-diffusively stable flames experiencing high positive curvature, where the curvature is defined as $C=\nabla \cdot \vec{N}$, and positive values correspond to flame elements that are convex with respect to the fresh gases. The density-weighted displacement speed of an isosurface of the progress variable, $s_{d}$, can be expressed as $s_{d} \rho_{0}|\nabla c|=\dot{\omega}_{c}+\vec{N} \cdot \nabla\left(\rho D_{c} \vec{N} \cdot \nabla c\right)-D_{c} C$, where $\dot{\omega}_{c}$ is the reaction rate, $D_{c}$ is the molecular diffusivity of the progress variable and $\rho_{0}$ is the density in the fresh gases. The last term on the right hand side represents the tangential diffusion of $c$, and for each isosurface of $c$ is directly proportional to curvature. Under 
conditions of large positive curvature this term can overcome the reactive and normaldiffusive contributions to produce negative, or counter-normal flame propagation. In addition, this effect becomes more pronounced for Le $>1$ since the diffusion of heat away from the reaction zone also causes a reduction in the positive contribution from the reaction rate. Conversely, for Le $<1$, the reaction rate contribution is increased in areas of positive curvature due to the enhanced diffusion of fuel into the reaction zone. For thermo-diffusively unstable flames this always exceeds the negative effects of tangential diffusion, thus, as the Lewis number is reduced the occurrence of CTC and $\mathrm{CPB}$ type interactions becomes increasingly unlikely.

The position and distribution of various types of interactions in each of the flames is illustrated in Fig. 6. It should be noted that the intensity of the interaction activity is reflected only partially by the absolute numbers shown in Fig. 6, since the sampling periods used are different for each case. It can be seen from Fig. 6 that the majority of interactions occur within the central region for all the flames where the turbulence is close to isotropic as discussed in Section 4.1. Normal type interactions become more tightly clustered in this central region as the Lewis number decreases, and this is a reflection of the more compact flame brush of the low Lewis number flames. Figure 7 shows profiles of the generalised Flame Surface Density (FSD), $\Sigma_{g}=\overline{|\nabla c|}$, along the centre lines of the domains as a function of downstream position, $x$, and progress variable $\tilde{c}$. Figure 8 shows the total number of normal interactions occurring within bins of $x$ and $\tilde{c}$ for each of the flames. Good qualitative agreement can be seen between both the magnitude and form of the $\Sigma_{g}$ profiles and the position and intensity of CX type interactions as a function of $x$, suggesting that the occurence of CX type interactions in these flames is strongly dependent on the expected flame surface area within any given volume. In progress variable space the CX interactions are more evenly dis- 
tributed compared to the profiles of $\Sigma_{g}$, which peak at $\tilde{c}$ values between 0.2 and 0.3 . However, it should be noted that whereas $\Sigma_{g}$ incorporates all isosurfaces of $c$, the flame interactions are defined only on the $c=0.8$ isosurface for which the FSD peak will be closer to the burnt gas side of the flame brush, and this may account for some of the discrepancy.

Increasing Lewis number creates a greater likelihood of counter-normal type interactions, as discussed above, and these occur predominantly in the areas immediately downstream of the flame holders where the turbulence is more strongly anisotropic. The initial counter-normal interactions ( $\mathrm{CN}$ types) appear to be caused by pairs of counter-rotating vortices which are continually generated in the shear layers surrounding the flame holders, and which force flame elements together against their normal propagation direction. Subsequent counter-normal interactions (CTC and CPB) follow these initial interactions if sufficiently large values of positive curvature are formed, as discussed above. This causal link between the counter-normal interaction types suggests a similar cascade relation between the interaction types that was previously proposed for normal type interactions (Dunstan et al., 2012), although the limited number of counter-normal interactions make this difficult to verify.

\subsection{Delay Time}

The cascade behaviour of normal type interactions can be clearly seen in Fig. 8. Distinct peaks in the distributions of CX and TC type interactions are visible, however, for flames B-C many of the fresh gas pockets that lead to PB type interactions are convected out of the downstream boundary before interaction can occur. It can be seen that for each of the flames the peaks of $\mathrm{CX}$ and $\mathrm{TC}$ interaction do not coincide, either 
in physical space or progress variable space. This is indicative of the cascade type behaviour of normal interaction types, and which results from the loose causal links between the flame topologies associated with each interaction type. A characteristic delay time between the $\mathrm{CX}$ and TC interaction types, $\tau_{C X-T C}$, can be identified by calculating the mean convection time between the limits $x 1$ and $x 2$ along the centre of the

domain, $\tau_{C X-T C} \approx \int_{x 1}^{x 2} d x / \tilde{u}_{x}$, where $x 1$ and $x 2$ are the mean streamwise positions for CX and TC type interactions respectively. Using this method gives $\tau_{C X-T C}=104,105$, 85 , and $47 \mu s$ for the flames A-D respectively. This compares well with the time of $71 \mu s$ obtained by Dunstan et al. (2012) for a unity Lewis number flame at comparable turbulence intensity using an alternative technique. The results appear to suggest that the delay time reduces with increasing laminar flame speed, however, the values for the flames $\mathrm{C}$ and D may be skewed by the fact that some TC interactions do not occur within the computational domains, and which may cause the second integral limit, $x 2$, to be unrealistically low.

\subsection{Interaction Length Scales}

The AFE technique outlined in Section 3 produces two quantities that can be used to identify characteristic length scales for the interactions: the change in the volume of burnt gas, $\Phi$, associated with each interaction, and the flame surface area enclosing this volume, $\phi$, which is equal to the sum of flame areas $\phi_{i}$ and $\phi_{i+1}$ associated with the time steps $t_{i}$ and $t_{i+1}$, as described in Section 3. From these the volume-based length scale, $L_{i n t}^{V}=\Phi^{1 / 3}$, and surface-area based length scale, $L_{i n t}^{S}=\phi^{1 / 2}$ can be defined, where $L_{i n t}^{V}$ is always less than $L_{i n t}^{S}$. The ratio $\Theta=L_{i n t}^{V} / L_{i n t}^{S}$ also provides information on the sphericity of the extracted regions and can take values in the range $0<\Theta \lesssim 0.455$, with the maximal value reached only for a perfect sphere. Mean values of $L_{i n t}^{S}$ and $\Theta$ are given in Table 3 for all the interaction types. Probability density functions illustrating 
the distribution of $L_{\text {int }}^{S}$ for CX and TC type interactions are shown in Fig. 9.

From the data in Table 3 and distributions shown in Fig. 9 it can be seen that, for the conditions considered in this study, the interaction length scales do not show any strong sensitivity to changes in the reactant Lewis number, and no significant difference in length scales exists between any of the sub-unity Lewis number flames. However, a small but significant reduction in both $L_{C X}^{S}$ and $L_{T C}^{S}$ can be seen for the super-unity Lewis number flame D. In addition, a small increase in $L_{C N}^{S}$ can also be seen in Flame D compared to flames B and C. These meaning of these results is discussed in section 4.5 in the context of the observed changes in flame area.

Counter-normal interactions have shorter length scales compared to normal type interactions, reflecting the longer time scales over which counter-normal interactions occur. The length scale ratio $\Theta$ remains almost constant across all interaction types with the exception of PB type interactions which show a strong tendency to approach the spherical limit for all the flames. It is notable that this is also true for the thermodiffusively unstable Flame A, where the tendency for flame elements to minimise their curvature, and therefore form spherical pockets, is not present. This suggests that thermo-diffusive effects may play only a secondary role in causing the spherical pocket shape and that the primary cause of due to the mechanism of their formation: where multiple TC type events occuring in close proximity have a tendency to produce approximately spherical pockets, which burn-out before thermo-diffusive effects have time to act.

\subsection{Flame Stretch}

The effects of flame interactions on the turbulent flame brush can best be understood by considering the stretch rates experienced by the flame from two different sources: turbulent processes (including straining and curvature) and changes in flame area due 
to flame interactions. The total global stretch rate can be written $K_{T O T}=(d A / d t) / A$, where $A$ is the surface area of the $c=0.8$ isosurface within the sampling region indicated in Fig. 1. This can also be written as a sum of flamelet, $K_{F}$ (representing all non-interacting turbulent processes), and interaction, $K_{I N T}$, contributions. The total stretch and interaction stretch contributions over the sample interval $\Delta t$ are given by:

$$
K_{T O T}=\frac{2\left(A_{i+1}-A_{i}\right)}{\left(A_{i+1}+A_{i}\right) \Delta t}
$$

and

$$
K_{I N T}=\frac{2\left(\phi_{i+1}-\phi_{i}\right)}{\left(A_{i+1}+A_{i}\right) \Delta t}
$$

where $A_{i}$ and $A_{i+1}$ are the total isosurface areas within the sampling region at times $t_{i}$ and $t_{i+1}$ respectively, and $\Delta \phi=\phi_{i+1}-\phi_{i}$ is the total change in area due to interactions occurring within the interval $\Delta t$, which is obtained using the AFE process outlined in Section 3. The flamlet contribution may be obtained from $K_{F}=K_{T O T}-K_{I N T}$. Interaction stretch may also be further decomposed by type, where: $K_{I N T}=\sum K_{\alpha}$, where $\alpha=$ CX, TC, PB, CN, CTC, and CPB.

The first task in assessing the contribution of flame interactions to the overall stretch rate is to examine the sensitivity of $K_{I N T}$ to the choice of the sample interval $\Delta t$. In general the flame interaction process includes multiple time scales, and thus it is clear that the change in area, $\Delta \phi$, extracted using AFE will strongly depend on the interval chosen for the analysis. By considering $K_{I N T}$ instead, $\Delta t$ is included in the denominator and so some of this dependence is taken into account. To determine what sensitivity remains in $K_{I N T}$, the AFE technique has been applied to the same data (flame C) using three equally spaced values for $\Delta t: a / 2, a$, and $3 a / 2$, where $a=16 \mu s$. The results are compared in Fig. 10 which shows the total stretch, $K_{T O T}$, the convex- 
normal (CX) interaction stretch, $K_{C X}$, and the tunnel-closure (TC) interaction stretch $K_{T C}$ for each value of $\Delta t$. It can be seen from Fig. 10 that $K_{T O T}$ shows very little sensitivity to $\Delta t$ and Eq. 2 acts as a de facto low-pass filter as $\Delta t$ is increased. However, the magnitude and form of $K_{T O T}$ are in good agreement for all $\Delta t$. In contrast, both $K_{C X}$ and $K_{T C}$ show significant variation with $\Delta t$. It is important to note that this is not due to errors in the AFE process itself since the interactions identified at each value of $\Delta t$ are essentially the same ${ }^{1}$, but it is in fact an intrinsic feature of the interaction stretch rates. For both $\mathrm{CX}$ and TC type interactions a general trend of increased magnitude of stretch rates with $\Delta t$ is apparent. For CX interactions, a variation in both the magnitude and sign of the stretch rates can also be seen.

To quantify this apparent sensitivity and to understand its root causes, it is useful to consider the mean and rms values of the stretch rates, given by: $\bar{K}=\left(1 / t_{s i m}\right) \int_{t 1}^{t 2} K d t$, and $K^{r m s}=\sqrt{\left[1 / t_{s i m} \int_{t 1}^{t 2} K^{2} d t\right]}$ respectively, where $t_{\text {sim }}$ is the total simulation time. These are listed in Table 4 for each value of $\Delta t$. As expected, the mean and rms values of $K_{\text {TOT }}$ remain similar for all $\Delta t$. For $K_{I N T}$, however, both the mean and rms values increase significantly with increasing $\Delta t$. The last two columns in Table 4 suggest that both the mean and rms values increase almost linearly with $\Delta t$, since dividing them by the sample interval removes much of the sensitivity. The reason for this can be understood by considering that the change in area resulting from the flame interactions over a given interval arises from two factors: differences in the magnitude of the area change for each interaction, and differences in the total number of interactions occurring within the interval. By working with the stretch rate rather than the area change, only one of these factors is taken into account. It is interesting that both of

\footnotetext{
${ }^{1}$ The numbers of interactions identified are 196, 192, and 192 with increasing $\Delta t$. The missing interactions are a result of two interaction types occurring in very rapid succession at the same location. This is unavoidable to some degree but constitutes only $2 \%$ of the total and therefore does not affect the analysis.
} 
these process appear to be approximately linear with respect to time on average over the range of sample intervals considered here. While it might reasonably be expected that the average number of interactions increases linearly with time, the area change due to individual interactions is, in general, a highly non-linear process which would normally preclude such a simple relationship.

The above observations highlight the importance of $\Delta t$ in the current analysis. While the actual point of interaction may be precisely located in time and space, the effect of each interaction on the subsequent flame stretch rate persists for some time following the interaction while the flame cusps recover from the extreme curvatures generated. So while it may be possible to identify a characteristic value for the rate of change of stretch due to interactions for a particular composition and turbulence level, the actual contribution from interactions is effectively defined by the choice of $\Delta t$. A value of $\Delta t$ must therefore be chosen that successfully separates the changes in area that arise from turbulent straining and curvature from those that could not occur through normal turbulent processes. This appears to introduce a degree of subjectivity into the assessment of the interaction stretch rates, however, it has been demonstrated by Chen et al. (1999) that a range of time scales for the interaction of lean premixed methane-air flames can be clearly identified, and which are independent of the time scales of the surrounding turbulence. Such an analysis forms a reasonable basis from which to constrain the range of appropriate values for $\Delta t$. The exact value will depend on the specific type of information required, for example the current range of $\Delta t$ ( $8 \leq \Delta t \leq 24 \mu \mathrm{s}$ ) spans the time scales identified by Chen et al. (1999) over which $95 \%$ of the increases in heat release rate and the flame displacement speed occur during interaction, and is therefore the most appropriate range for analysing the heat release rate in turbulent flames with flame-flame interactions. 
Considering the effects of Lewis number on the flame stretch, Figure 11 shows the total, flamelet, and interaction stretch rates for all flames in the database. A clear trend of lower stretch rates of all types with increasing thermo-diffusive stability or Lewis number can be seen. This is most noticeable in the thermo-diffusively unstable flame A, where self-induced enhancement of stretch rates in positively curved regions causes larger fluctuations in $K_{F}$ than could be achieved by turbulent processes alone. These differences are quantified in Table 5 which shows the mean and rms values of the stretch rates for all flames by interaction type. It should be noted that since the flame is stationary, in the limit of $T \rightarrow \infty, \bar{K}_{T O T}$ should approach zero and $\bar{K}_{F}$ and $\bar{K}_{I N T}$ should balance. This is approximately so for all the flames, although a slight lack of statistical convergence is evident for the flame $\mathrm{C}$.

The following general observations can be made about Fig. 11 and the data in Table 5: In agreement with previous findings, CX type interactions can lead to either positive or negative individual stretch contributions but overall their contribution is always negative for the flames investigated here. TC type interactions are responsible for the largest overall negative stretch rates in all the cases, and are also almost always negative individually. PB type interactions are the second largest cause of negative interaction stretch rates; they are always negative but occur less frequently than other normal type interactions, even when the loss of fresh gas pockets through the downstream boundary is taken into account. The rms values of the interaction stretch contributions range between approximately $18 \%$ and $45 \%$ of the flamelet stretch contributions (for $\Delta t=16 \mu \mathrm{s}$ ).

As the Lewis number increases the contribution from all normal type interactions decreases. Conversely, the contribution from all counter-normal interaction types increases with increasing Lewis number. It should also be noted that the differences in $\overline{K_{C X}}$ and $\overline{K_{T C}}$ between the flames $\mathrm{B}$ and $\mathrm{C}$, and between the flames $\mathrm{C}$ and $\mathrm{D}$ are ap- 
proximately the same, whereas the values for the flame A are an order of magnitude greater, indicating a qualitative change in behaviour for the flame A.

The changes in $\overline{K_{\alpha}}$ magnitude can be understood as the product of changes in the frequency of interactions, $f_{\alpha}=N_{\alpha} / t_{\text {sim }}$, where $N_{\alpha}$ is the total number of interactions of type $\alpha$, and $t_{\text {sim }}$ is the simulation time, and changes in the mean stretch rate per interaction, $\overline{K_{\alpha}^{i}}=\left(1 / N_{\alpha}\right) \sum K_{\alpha}$. The time-averaged stretch rate is then recovered from the relation $\overline{K_{\alpha}}=\overline{K_{\alpha}^{i}} f_{\alpha} \Delta t$. These quantities have been calculated for all TC events in all the flames considered and the results are given in Table 6.

From the data in Table 6 it can be seen that both $\overline{K_{T C}^{i}}$ and $f_{T C}$ are equally important in determining the changes in overall interaction stretch contributions. Thus, sensitivity to Lewis number manifests itself both in large-scale factors such as the number of interactions occurring, which is related to the flame surface density as demonstrated in Section 4.2, and also on the fine-scale details of the individual interaction events. It is interesting to compare the sensitivity of $\overline{K_{T C}^{i}}$ to Lewis number with the observation made in section 4.4 that the value of the mean length scale $\overline{L_{i n t}^{S}}$ is approximately constant for normal type interactions regardless of Lewis number. Taken together these observations show that while the total flame area associated with each normal interaction remains similar for all the flames, the proportion of that flame area associated with pre-interaction and post-interaction flame elements is significantly affected by Lewis number. The cause of this can be traced to differences in the magnitude of curvature and the degree of alignment of the flame elements prior to merger for each of the flames. For example, flame elements that are weakly curved and therefore aligned over a wider area prior to interaction will produce a larger reduction in flame area than those that are more strongly curved prior to interaction. Low Lewis number flames exhibit a tendency to form more sheet like flame elements interspersed with ridge-like protrusions into the fresh gases (Bell et al., 2013). This leads to a greater number of 
interactions between weakly curved flame elements, which, due to the more extensive alignment of the flame elements under these conditions, results in a greater reduction in flame area.

These results also suggest that, because of the scale-separation between the interaction time scales and thermo-diffusive time scales, Lewis number effects play only a minor role during the flame interactions. However, large scale changes in flame wrinkling, induced by the thermo-diffusive effects acting over longer time scales, produce significant changes both in the magnitude and frequency of interactions for different Lewis numbers.

For the purposes of modelling it is useful to consider whether the observed variations in $\overline{K_{T C}^{i}}$ and $f_{T C}$ can be related to the thermo-chemical properties of the flames listed in Table 1. Due to the partial decoupling of temperature and fuel mass fraction profiles for non-unity Lewis number flames, there are several length and time scales available for the normalisation of $\overline{K_{T C}^{i}}$ and $f_{T C}$, and the correct scaling parameter may not be the same for both Le $<1$ and Le $>1$ flames due to the qualitative changes in flame structure in these flames. This indeed appears to be the case for the flames investigated here. As shown in Table 6, a reasonable collapse of the data can be achieved using the time scale associated with the limiting diffusivity for each flame (i.e. $\tau_{t h} \equiv \delta_{t h} / s_{L}$ for Le $<1$, and $\tau_{c} \equiv \delta_{c} / s_{L}$ for Le $>1$ ). The Damköhler number in Table 6 is defined as $\widehat{\mathrm{Da}}=\left(l_{0 i n} / u_{\text {in }}^{\prime}\right) / \min \left(\tau_{c}, \tau_{t h}\right)$, the normalised stretch rate is $\hat{K}_{T C}^{i}=\bar{K}_{T C}^{i}\left(l_{0 i n} / u_{i n}^{\prime}\right) \widehat{\mathrm{Da}}$, and the normalised frequency is $\hat{f}_{T C}=f_{T C}\left(l_{0 i n} / u_{i n}^{\prime}\right) \widehat{\mathrm{Da}}$.

It is important to note that this scaling may not be unique and so does not necessarily indicate the underlying physical mechanism causing the observed differences. Indeed, a similar collapse of the data in Table 6 can also be achieved using the normalised Lewis number $\hat{L e}=L e /(L e+1)$, which is constructed by analogy with the 
normalised equivalence ratio suggested by Law (2006), and which removes the inherent asymmetry between Le $<1$ and Le $>1$ in the standard Lewis number.

\section{Conclusions}

The effects of non-equidiffusive transport on flame interactions in premixed flames have been investigated using high-resolution simulations of twin turbulent V-flames with global Lewis numbers of 0.4, 0.8, 1.0 and 1.2. Interactions were extracted using the AFE method described in Dunstan et al. (2012), and the sensitivity of the results to the choice of sample interval $\Delta t$ was assessed. It was found that both the average number of interactions occurring within the interval, as well as the mean magnitude of the area change associated with each interaction both increase approximately linearly with $\Delta t$ over the range considered, leading to an overall linear dependence of the interaction stretch rate with sample interval $\Delta t$. This occurs because while flame interactions can be located precisely in time and space, their effects on the subsequent flame evolution persist over some characteristic time scale associated with the cusp recovery. The sensitivity to $\Delta t$ is therefore not an artifact of the AFE method employed here, but reflects an inherent property of flame interactions that applies to any attempt to separate the effects of transient processes such as interaction stretch from the continuous turbulent processes in a flow.

In agreement with previous findings, normal type interactions occur in a cascade going from $\mathrm{CX} \rightarrow \mathrm{TC} \rightarrow \mathrm{PB}$, and with a characteristic delay time of the same order ( $0.1 \mathrm{~ms})$ as identified previously using a different method. For all flames TC type interactions cause the greatest loss of flame area, followed by PB types. CX interactions 
can individually lead to an increase or decreases in flame area but cause a net loss overall for all Lewis numbers. Three counter-normal interaction types were also identified, $\mathrm{CN}, \mathrm{CTC}$, and $\mathrm{CPB}$, which are analogous to the normal types but occur on the burnt-gas side of the flames.

Interaction length scales remain similar for unity and sub-unity Lewis number flames, but are slightly reduced for the super-unity Lewis number flame.

Variation in Lewis number affects flame interactions in the following principal ways:

- Lower Lewis numbers are associated with faster turbulent flame speeds and a more compact flame brush, which leads to a higher frequency of interactions and a more compact distribution of interactions in physical space. The distribution of CX type interactions in mean progress variable space, $\tilde{c}$, remains unaffected, but TC type interactions peak at lower values of $\tilde{c}$ for increasing Lewis number, due to the greater thickness of the flame brush with increasing Le.

- Higher Lewis number flames show a greater propensity for counter-normal type interactions. CTC and CPB types are only observed in Flame D, and no counternormal interactions occur in Flame A. The occurrence of CTC and CPB types depends on the existence of counter-normal flame displacement speeds, which become more likely at higher Lewis numbers.

- Global stretch rates due to turbulent strain and curvature, and due to flames interactions both increase with decreasing Lewis number. Mean and rms stretch rate values show similar qualitative trends, with the thermo-diffusively unstable Flame A showing the largest increases. 
- Changes in both the frequency of interactions, and in the mean magnitude of individual interactions are equally important in producing the changes in overall interaction stretch contributions. Both the frequency and the mean magnitude scale well with the inverse of the laminar flame time scale associated with the limiting diffusivity of each flame, as well as the normalised Lewis number.

This last observation is particularly interesting, since it might be anticipated that the longer flame time scales and slower flame speeds associated with the lower Lewis number flames would result in a decrease in the change in flame area following interaction over a fixed interval $\Delta t$, and hence a reduction in the mean magnitude of individual interactions. This is particularly the case when one considers the kinematics of curved, non-unity Lewis number flames. The displacement speed of negatively curved flame elements increases with Lewis number, and for thermo-diffusively unstable flames negative displacement speeds are possible. For CX and TC type interactions, very large negative curvatures are produced at the point of interaction, and so it might be expected that the rate of cusp recovery - and hence the rate of area change - for lower Lewis number flames should be significantly less than for high Lewis number flames.

The fact that the results indicate the exact opposite of this trend prompts the following conclusions: a) Since normal type flame interactions occur over time scales that are very much shorter than either the thermal or mass diffusive time scales of the flames, Lewis number effects do not significantly influence the interaction process itself. b) The changes observed in the frequency and magnitude of interactions with Lewis number can both be attributed to the large-scale changes in flame structure for each of the flames. The increased flame area associated with lower Lewis numbers causes an increase in the frequency of interactions, whereas changes to the local ge- 
ometry of the flames (the curvature and degree of alignment of flame elements prior to merger) determines the magnitude of the area change for each interaction. c) Counternormal interactions occur over longer time scales and so are more sensitive to changes in the kinematics of the flames, as well as large scale changes in flame structure.

Confirmation of these conclusions will require further investigation, however, the apparent insensitivity of individual interactions to diffusive processes is in agreement with the observations by Sung and Law (2000) on the attenuation of the flame response to high frequency fluctuations in stretch rate. The existence of a degree of time scale separation between these processes suggests some potentially useful model simplifications.

\section{Acknowledgments}

The authors would like to thank Professor R. S. Cant for the use of SENGA2. EPSRC funding through grant number EP/F028741/1, and funding from Rolls-Royce is acknowledged.

\section{References}

R. G. Abdel-Gayed, D. Bradley, M. N. Hamid, and M. Lawes. Lewis number effects on turbulent burning velocity. Proc. Combust. I., 1:505-512, 1984.

R. Balachandran, B. O. Ayoola, C. F. Kaminski, A. P. Dowling, and E. Mastorakos. Experimental investigation of the nonlinear response of turbulent premixed flames to imposed inlet velocity oscillations. Combust. Flame, 143:37-55, 2005.

J. B. Bell, R. K. Cheng, M. S. Day, and I. G. Shepherd. Numerical simulation of lewis number effects on lean premixed turbulent ames. Proc. Combust. I., 31:1309-1317, 2007. 
J.B. Bell, M.S. Day, and M.J. Lijewski. Simulation of nitrogen emissions in a premixed hydrogen flame stabilized on a low swirl burner. Proc. Combust. Inst., 34:1173$1182,2013$.

S. Candel, D. Durox, and T. Schuller. Flame interactions as a source of noise and combustion instabilities. AIAA, 2004-2928, 2004.

C. L. Chen and S. H. Sohrab. Upstream interactions between planar symmetric laminar methane premixed flames. Combust. Flame, 101:360-370, 1995.

H. Chen and N. G. Kingsbury. Efficient registration of non-rigid 3-D bodies. IEEE T. Image Process., 21:262 - 272, 2012.

J. H. Chen, T. Echekki, and W. Kollmann. The mechanism of two-dimensional pocket formation in lean premixed methane-air flames with implications to turbulent combustion. Combust. Flame, 116:15-48, 1999.

T. D. Dunstan, N. Swaminathan, and K. N. C. Bray. Geometrical properties and turbulent flame speed measurements in stationary premixed V-flames using direct numerical simulations. Flow Turbul. Combust., 87:237-259, 2011.

T. D. Dunstan, N. Swaminathan, K. N. C. Bray, and N. G. Kingsbury. Flame interactions in turbulent premixed twin v-flames. Combust. Sci. and Technol., In Press, 2012.

P. J. Goix and I. G. Shepherd. Lewis number effects on turbulent premixed flame structure. Combust. Sci. Technol., 91:191-206, 1993.

I. R. Gran, T. Echekki, and J. H. Chen. Negative flame speed in an unsteady 2-D premixed flame: a computational study. Proc. Combust. I., 26:323-329, 1996. 
E. R. Hawkes and J. H. Chen. Direct numerical simulation of hydrogen-enriched lean premixed methane-air flames. Combust. Flame, 138:242-258, 2004.

D. C. Haworth and T. J. Poinsot. Numerical simulations of lewis number effects in turbulent premixed flames. J. Fluid. Mech., 244:405-436, 1992.

K. W. Jenkins and R. S. Cant. DNS of turbulent flame kernels. In Proceedings of the Second AFOSR Conference on DNS and LES, pages 192-202, 1999.

B. Jones. Application of lean flames in stationary gas turbuines. In N. Swamianthan and K.N.C. Bray, editors, Turbulent Premixed Combustion, pages 309-351. Cambridge University Press, 2011.

N. G. Kingsbury. Complex wavelets for shift invariant analysis and filtering of signals. J. Appl. Comput. Harmon. Anal., 10:3:234-253, 2001.

W. Kollmann and J. H. Chen. Pocket formation and the flame surface density equation. Proc. Combust. I., 27:927-934, 1998.

L. W. Kostiuk, I. G. Shepherd, and K. N. C. Bray. Experimental study of premixed turbulent combustion in opposed streams. part III: spatial structure of flames. Combust. Flame, 118:129-139, 1999.

C. K. Law. Combustion Physics. Cambridge University Press, 2006.

C. K. Law and C. J. Sung. Structure, aerodynamics, and geometry of premixed amelets. Prog. Energ. Combust. Sci., 26:459-505, 2000.

S. D. Lee and S. H. Chung. Experimental investigation of the nonlinear response of turbulent premixed flames to imposed inlet velocity oscillations. Combust. Flame, 98:80-92, 1994. 
A. N. Lipatnikov and J. Chomiak. Molecular transport effects on turbulent flame propagation and structure. Prog. Energ. Combust. Sci., 31:1-73, 2005.

B.J. McBride, S. Gordon, and M.A. Reno. Coefficients for calculating thermodynamic and transport properties of individual species. NASA Technical Report, TM-4513, 1993.

N. Peters. Turbulent Combustion. Cambridge University Press, 2000.

S. B. Pope. Turbulent Flows. Cambridge University Press, 2000.

T. Schuller, D. Durox, and S. Candel. Dynamics of and noise radiated by a perturbed impinging premixed jet flame. Combust. Flame, 128:88-110, 2002.

S. H. Sohrab, Z. Y. Lee, and C. K. Law. An experimental investigation on flame interaction and the existence of negative flame speeds. Proc. Combust. Inst., 20: 1957-1965, 1984.

C. J. Sung and C. K. Law. Structural sensitivity, response, and extinction of diffusion and premixed flames in oscillating counterflow. Combust. Flame, 123:375-388, 2000.

M. Talei, M. J. Brear, and E. R. Hawkes. A parametric study of sound generation by premixed laminar flame annihilation. Combust. Flame, 159:757-769, 2012. 
Table 1: Thermo-chemical parameters. For all flames $T_{i n}=600 \mathrm{~K}$, and $\tau=\left(T_{a d}-\right.$ $\left.T_{\text {in }}\right) / T_{\text {in }}=2.52$

\begin{tabular}{lccccccc}
\hline Case & Le & $s_{L}\left(\mathrm{~ms}^{-1}\right)$ & $\delta_{\text {th }}(\mathrm{mm})$ & $\delta_{c}(\mathrm{~mm})$ & $\delta(\mathrm{mm})$ & $\tau_{\text {th }}(\mathrm{ms})$ & $\tau_{c}(\mathrm{~ms})$ \\
\hline A & 0.4 & 0.420 & 0.620 & 0.863 & 0.434 & 1.476 & 2.054 \\
B & 0.8 & 0.558 & 0.464 & 0.482 & 0.163 & 0.832 & 0.864 \\
C & 1.0 & 0.603 & 0.430 & 0.430 & 0.121 & 0.713 & 0.713 \\
D & 1.2 & 0.639 & 0.403 & 0.375 & 0.095 & 0.631 & 0.586 \\
\hline
\end{tabular}

Table 2: Turbulence parameters at the inlet. For all flames $\operatorname{Re}_{l_{0}}=82$ and $\bar{u}_{\text {in }}=10$ $\left(\mathrm{ms}^{-1}\right)$.

\begin{tabular}{lcccc}
\hline Case & $u_{i n}^{\prime} / s_{L}$ & $l_{0, i n} / \delta_{c}$ & $K a$ & $D a$ \\
\hline A & 11.6 & 0.99 & 28.3 & 0.09 \\
B & 8.76 & 1.77 & 11.3 & 0.20 \\
C & 8.10 & 1.94 & 8.67 & 0.24 \\
D & 7.65 & 2.28 & 7.06 & 0.29 \\
\hline
\end{tabular}

Table 3: Mean interaction length scale $\overline{L_{\text {int }}^{S+}}=\overline{\phi^{1 / 2}}$ and sphericity ratio $\Theta$ by type for all flames.

\begin{tabular}{lcccccccccccc}
\hline Case & $L_{C P B}^{S+}$ & $L_{C T C}^{S+}$ & $L_{C N}^{S+}$ & $L_{C X}^{S+}$ & $L_{T C}^{S+}$ & $L_{P B}^{S+}$ & $\Theta_{C B P}$ & $\Theta_{C T C}$ & $\Theta_{C N}$ & $\Theta_{C X}$ & $\Theta_{T C}$ & $\Theta_{P B}$ \\
\hline A & - & - & - & 3.46 & 3.46 & 2.36 & - & - & - & 0.33 & 0.34 & 0.42 \\
B & - & - & 1.81 & 3.48 & 3.38 & 2.19 & - & - & 0.37 & 0.34 & 0.35 & 0.43 \\
C & - & - & 1.83 & 3.42 & 3.43 & 2.76 & - & - & 0.37 & 0.34 & 0.35 & 0.41 \\
D & 2.39 & 1.95 & 2.04 & 3.21 & 3.12 & 2.36 & 0.34 & 0.37 & 0.36 & 0.35 & 0.36 & 0.43 \\
\hline
\end{tabular}

Table 4: Sensitivity of mean and rms global stretch rates $\left(\mathrm{s}^{-1}\right)$ to sample interval, $\Delta t$, for flame C. $a=16 \mu \mathrm{s}$.

\begin{tabular}{lcccccc}
\hline$\Delta t$ & $\bar{K}_{T O T}$ & $K_{T O T}^{r m s}$ & $\bar{K}_{I N T}$ & $K_{I N T}^{r m s}$ & $\bar{K}_{I N T} / \Delta t$ & $K_{I N T}^{r m s} / \Delta t$ \\
\hline$a / 2$ & -34 & 588 & -38 & 79 & $-76 / a$ & $157 / a$ \\
$a$ & -31 & 583 & -77 & 126 & $-77 / a$ & $126 / a$ \\
$3 a / 2$ & -32 & 576 & -113 & 173 & $-75 / a$ & $116 / a$ \\
\hline
\end{tabular}

Table 5: Mean and rms global stretch rates for all cases $\left(\mathrm{s}^{-1}\right)$.

\begin{tabular}{lccccccccccc}
\hline Case & $\bar{K}_{T O T}$ & $\bar{K}_{F}$ & $\bar{K}_{I N T}$ & $\bar{K}_{C X}$ & $\bar{K}_{T C}$ & $\bar{K}_{P B}$ & $\bar{K}_{C N}$ & $\bar{K}_{C T C}$ & $\bar{K}_{C P B}$ & $K_{F}^{r m s}$ & $K_{I N T}^{r m s}$ \\
\hline A & 13 & 317 & -304 & -26 & -204 & -74 & - & - & - & 876 & 392 \\
B & -13 & 81 & -94 & -9 & -74 & -11 & -1 & - & - & 586 & 144 \\
C & -31 & 46 & -77 & -5 & -56 & -14 & -2 & - & - & 555 & 126 \\
D & 7 & 57 & -51 & -1 & -35 & -5 & -6 & -4 & -0 & 507 & 91 \\
\hline
\end{tabular}


Table 6: Mean individual interaction stretch rate magnitudes and frequencies for TC events.

\begin{tabular}{lccccc}
\hline Case & $\bar{K}_{T C}^{i}\left(\mathrm{~s}^{-1}\right)$ & $f_{T C}(\mathrm{KHz})$ & $\widehat{\mathrm{Da}}\left(\times 10^{4}\right)$ & $\hat{K}_{T C}^{i}\left(\times 10^{6}\right)$ & $\hat{f}_{T C}\left(\times 10^{3}\right)$ \\
\hline $\mathrm{A}$ & -133 & 96 & 1.17 & -2.70 & 1.95 \\
$\mathrm{~B}$ & -89 & 52 & 2.08 & -3.20 & 1.87 \\
$\mathrm{C}$ & -80 & 44 & 2.43 & -3.36 & 1.85 \\
$\mathrm{D}$ & -57 & 38 & 2.95 & -2.91 & 1.94 \\
\hline
\end{tabular}




\section{List of Figures}

1 Two-dimensional slice of instantaneous progress variable, $c$ overlaid with contours of Favre averaged progress variable (black) at $\tilde{c}=0.1,0.2, \ldots, 0.9$ for flame C. Transverse profile positions indicated by thick dashed lines. Interaction zone where AFE is applied is indicated by rectan-

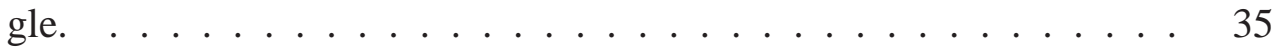

2 Global product mass fraction, $\Omega$, for all cases from initialisation at $t=0$. Dashed lines indicate start of data collection period for flames A-D (left to right respectively). . . . . . . . . . . 36

3 (a) Favre averaged streamwise velocity, $\tilde{u}$, along the domain centre line ( $\left.y=L_{y} / 2\right)$ for all cases; (b) transverse profiles of $\tilde{u}$ at downstream positions indicated in Fig. 1 for flame C; (c) transverse profiles of rms turbulent fluctuations, $u^{\prime}$, for flame C . . . . . . . . 36

$4 \quad$ Anisotropy invariants $\xi$ (left) and $\eta$ (right) for flame B. . . . . . . . 37

5 Schematic diagram of interaction types. Reactants and products are denoted R and P respectively. (a) CX; (b) TC; (c) PB. Three additional counter-normal types, CN, CTC, and CPB are topologically similar but with $\mathrm{R}$ and $\mathrm{P}$ reversed.

6 Contours of Favre averaged progress variable $(\tilde{c}=0.1,0.2, \ldots, 0.9)$ with positions of all interactions in the x-y plane for flames A-D ((a)(d) respectively). Types of interactions, shown in Fig. 5 and discussed in section 4.2, are marked as follows: red diamonds are CX; blue circles are TC; brown stars are PB; filled black squares are $\mathrm{CN}$; unfilled black circles are CTC; and unfilled black stars are CPB. . . . . . . . . 38 
7 Flame Surface Density profiles along the domain centre lines $(y=$ $L_{y} / 2$ ) for all cases as a function of (a) distance from the inlet, and (b) Favre averaged progress variable. . . . . . . . . . . . . . . 39

8 Number of interactions by type occuring within bins of width $L_{x} / 30$ for downstream distance $x$ (left column), and within bins of width 1/20 for Favre averaged progress variable $\tilde{c}$ (right column). . . . . . . . . . 39

9 Probability densities of interaction length scales for all cases: a) $L_{i n t}^{S+}$ for CX interactions, b) $L_{i n t}^{S+}$ for TC interactions. (Colour online) . . . . 40

10 Sensitivity of the AFE technique to interval size. Total stretch rate (top) and interaction stretch rates for CX (middle) and TC (bottom) types. Three intervals sizes, $\Delta t$, shown where $a=16 \mu \mathrm{s} . ~ \ldots \ldots . . . .40$

11 Stretch rates of the $c=0.8$ isosurface for cases flames A-D ((a)-(d) respectively). Top: total stretch rate, $K_{T O T}$, flamelet component, $K_{F}$ , and interactions component, $K_{I N T}$. Bottom: interaction stretch rates by type. The $x$-axes of figures a-d have been aligned so that conditions at the inlet are identical for each simulation at a given value of $t . \ldots$ 


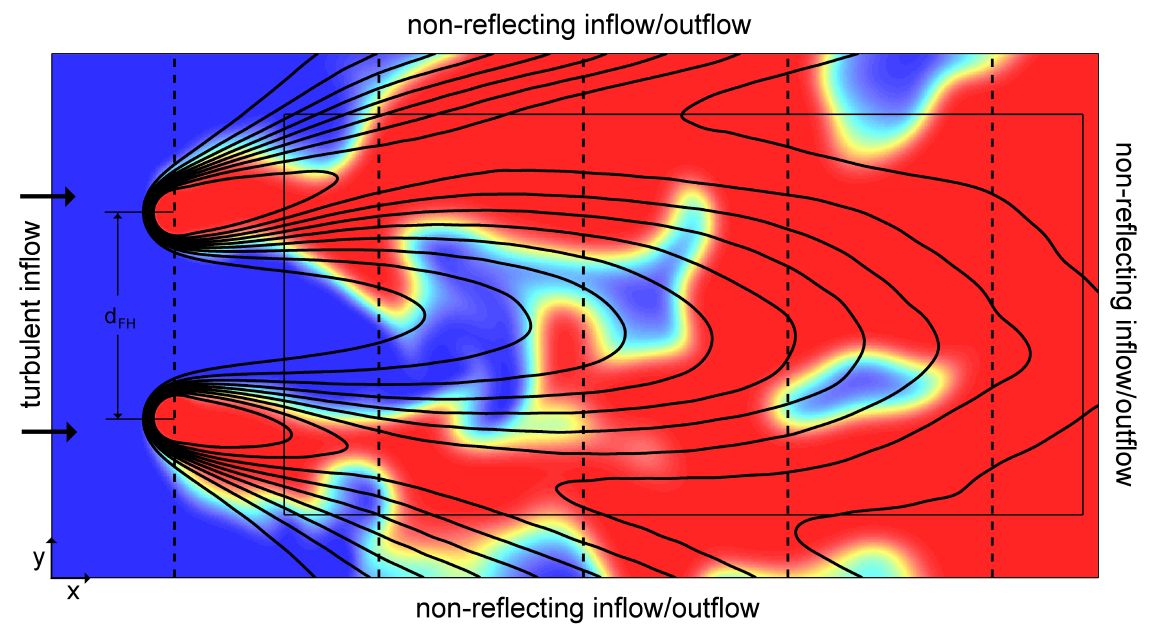

Figure 1: Two-dimensional slice of instantaneous progress variable, $c$ overlaid with contours of Favre averaged progress variable (black) at $\tilde{c}=0.1,0.2, \ldots, 0.9$ for flame C. Transverse profile positions indicated by thick dashed lines. Interaction zone where AFE is applied is indicated by rectangle. 


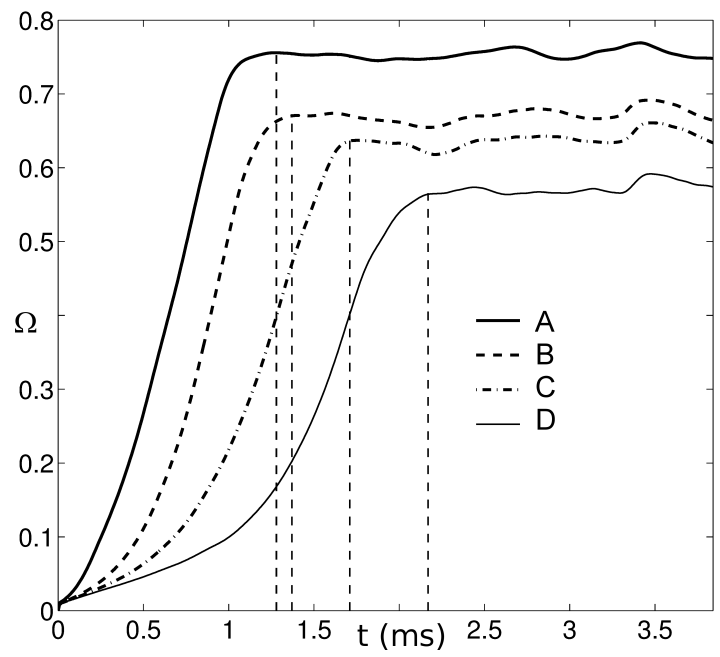

Figure 2: Global product mass fraction, $\Omega$, for all cases from initialisation at $t=$ 0 . Dashed lines indicate start of data collection period for flames A-D (left to right respectively).
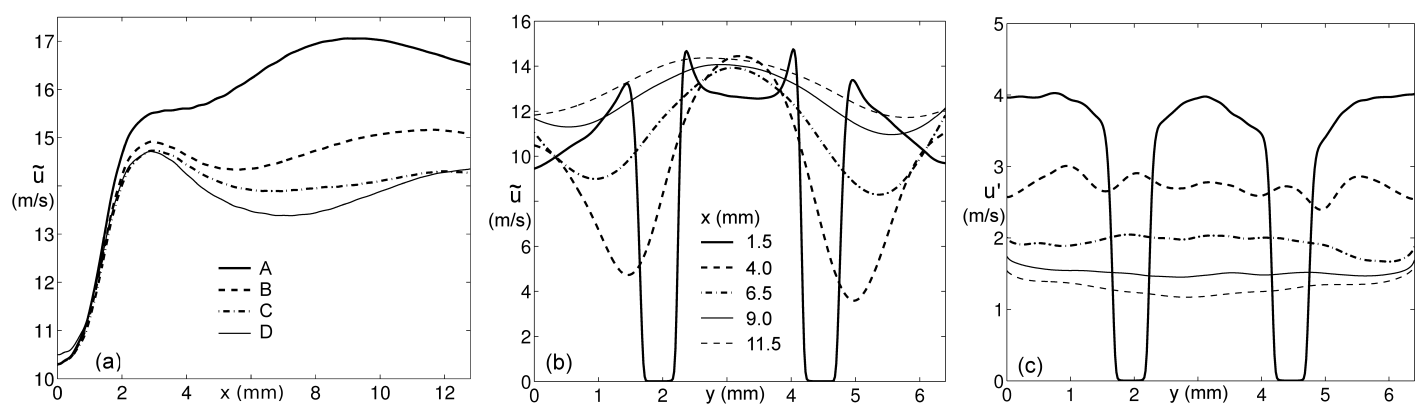

Figure 3: (a) Favre averaged streamwise velocity, $\tilde{u}$, along the domain centre line $\left(y=L_{y} / 2\right)$ for all cases; (b) transverse profiles of $\tilde{u}$ at downstream positions indicated in Fig. 1 for flame C; (c) transverse profiles of rms turbulent fluctuations, $u^{\prime}$, for flame C. 

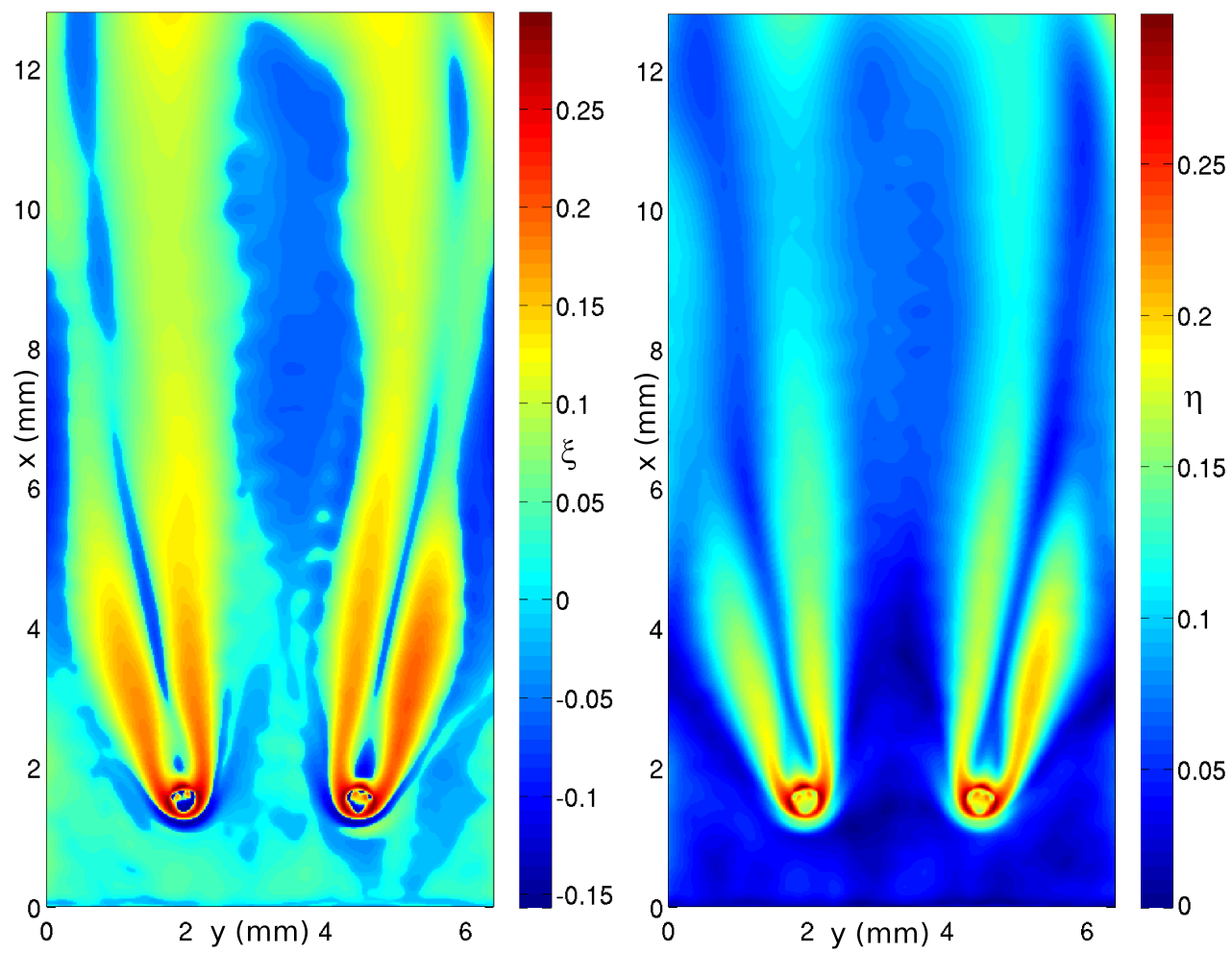

Figure 4: Anisotropy invariants $\xi$ (left) and $\eta$ (right) for flame B.

(a)

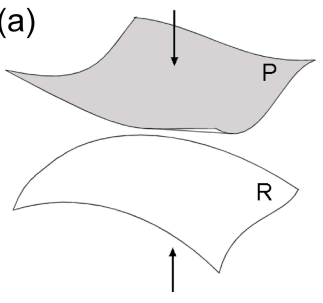

$\downarrow$

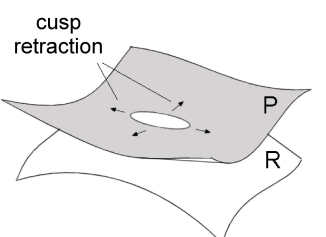

(b)

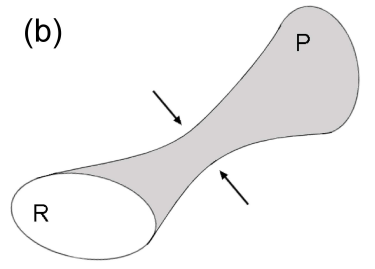

$\downarrow$

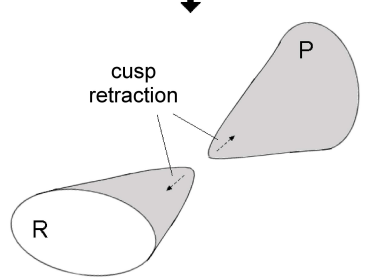

(c)

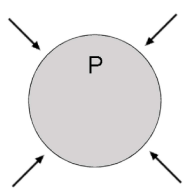

$\downarrow$

Figure 5: Schematic diagram of interaction types. Reactants and products are denoted $\mathrm{R}$ and $\mathrm{P}$ respectively. (a) CX; (b) TC; (c) PB. Three additional counter-normal types, $\mathrm{CN}, \mathrm{CTC}$, and CPB are topologically similar but with $\mathrm{R}$ and $\mathrm{P}$ reversed. 

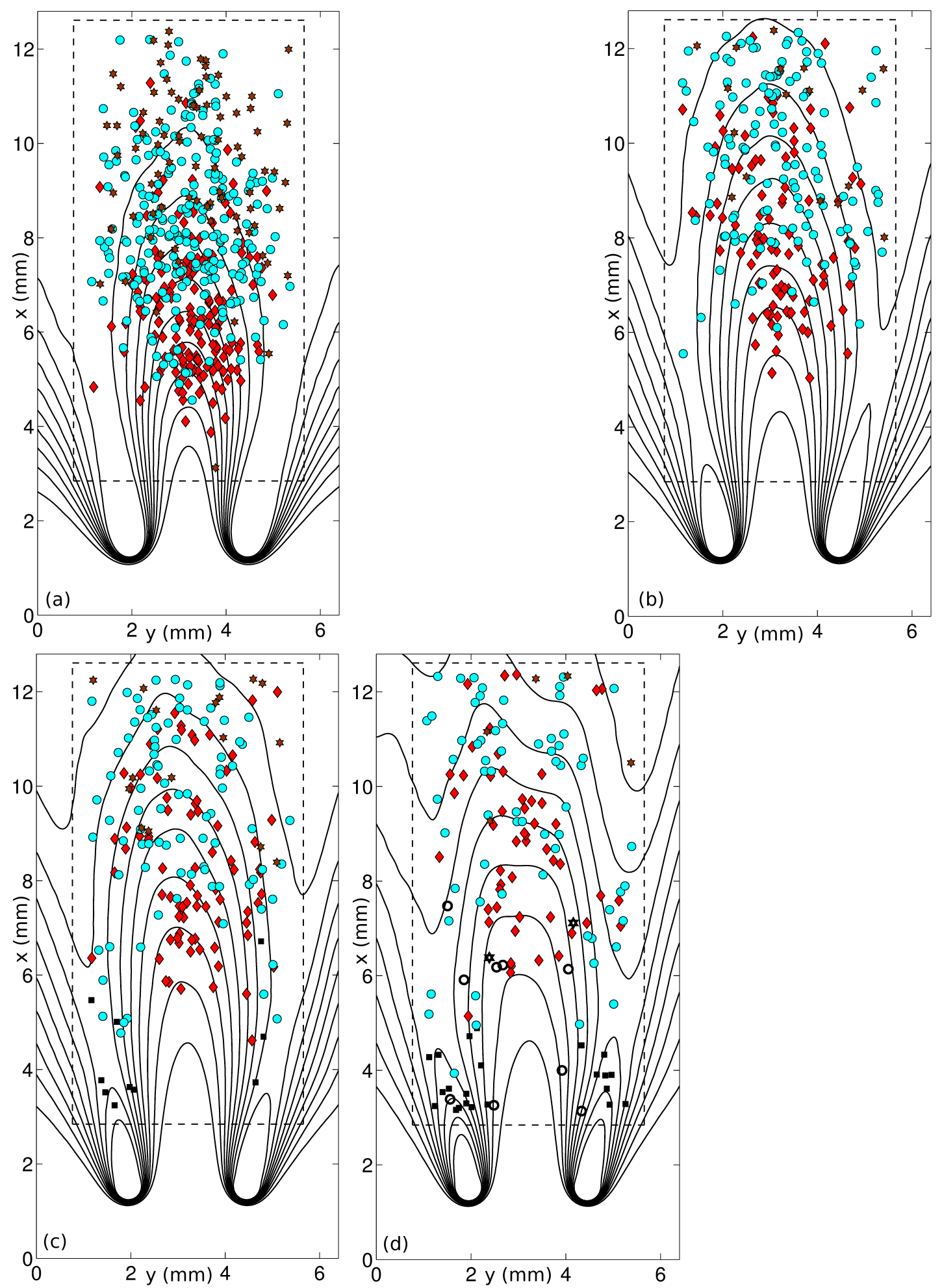

Figure 6: Contours of Favre averaged progress variable $(\tilde{c}=0.1,0.2, \ldots, 0.9)$ with positions of all interactions in the $x-y$ plane for flames A-D ((a)-(d) respectively). Types of interactions, shown in Fig. 5 and discussed in section 4.2, are marked as follows: red diamonds are CX; blue circles are TC; brown stars are $\mathrm{PB}$; filled black squares are $\mathrm{CN}$; unfilled black circles are CTC; and unglled black stars are CPB. 

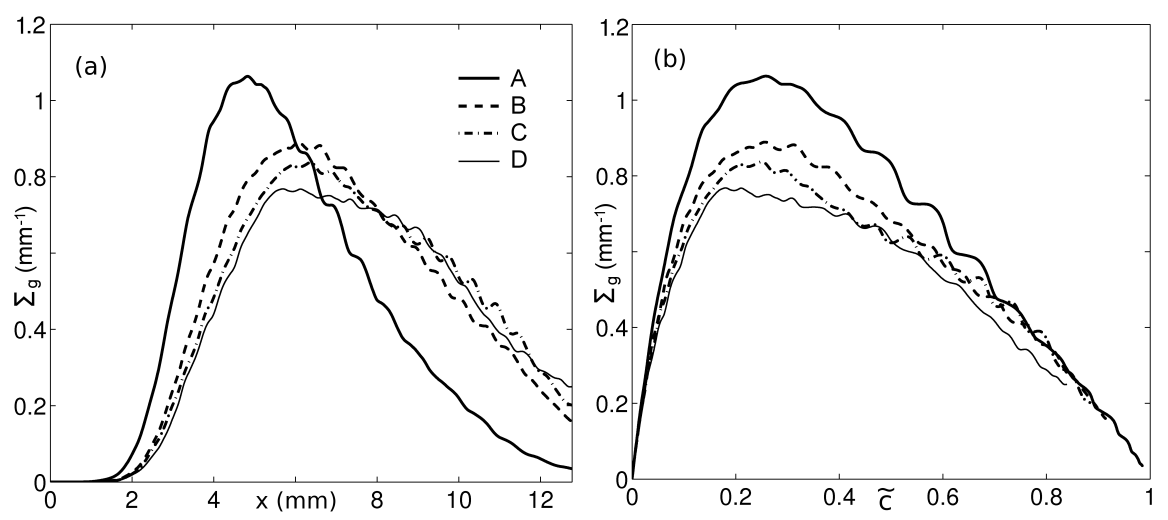

Figure 7: Flame Surface Density profiles along the domain centre lines $\left(y=L_{y} / 2\right)$ for all cases as a function of (a) distance from the inlet, and (b) Favre averaged progress variable.

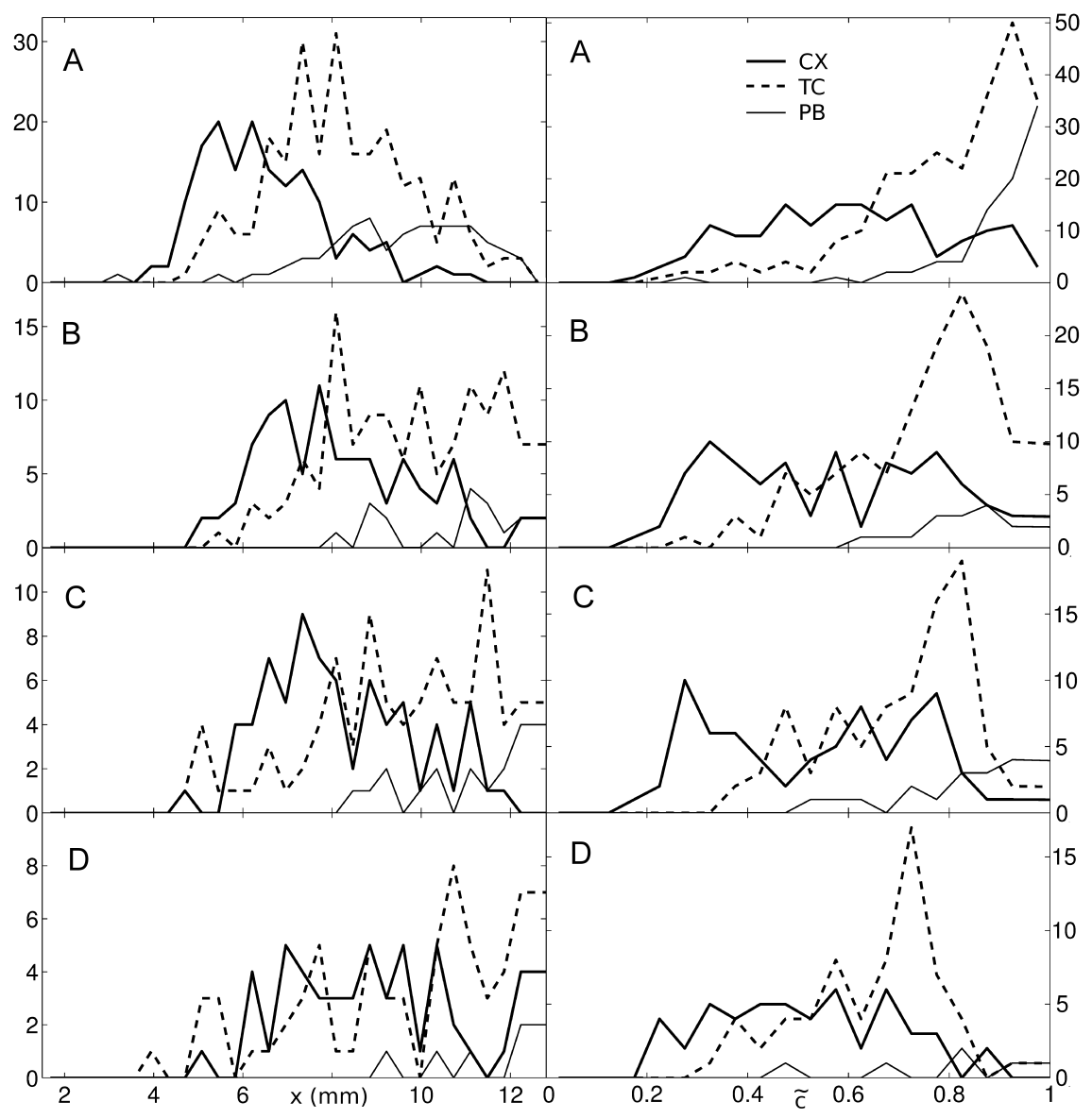

Figure 8: Number of interactions by type occuring within bins of width $L_{x} / 30$ for downstream distance $x$ (left column), and within bins of width 1/20 for Favre averaged progress variable $\tilde{c}$ (right column). 

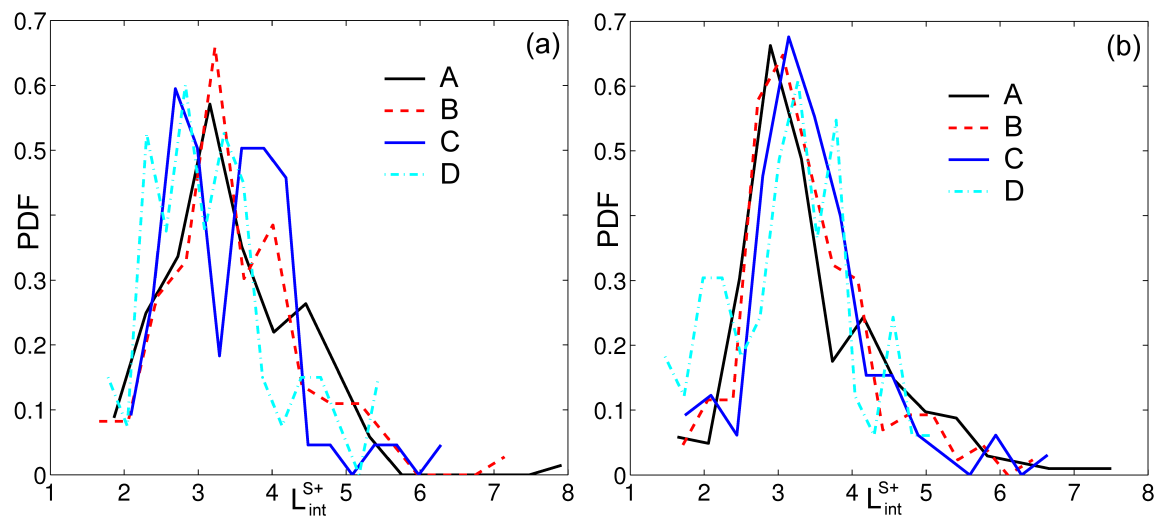

Figure 9: Probability densities of interaction length scales for all cases: a) $L_{\text {int }}^{S+}$ for CX interactions, b) $L_{i n t}^{S+}$ for TC interactions. (Colour online)

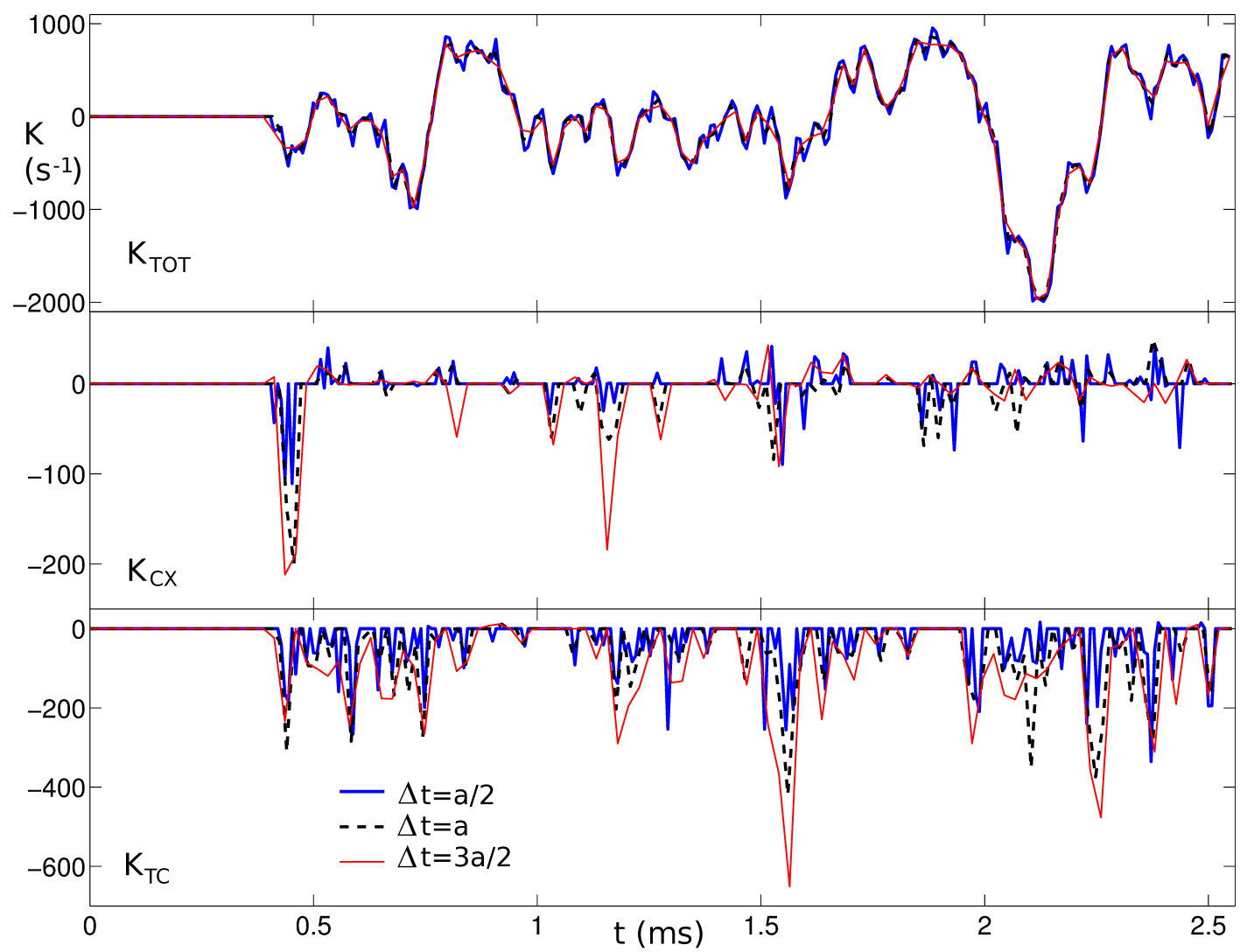

Figure 10: Sensitivity of the AFE technique to interval size. Total stretch rate (top) and interaction stretch rates for CX (middle) and TC (bottom) types. Three intervals sizes, $\Delta t$, shown where $a=16 \mu \mathrm{s}$. 

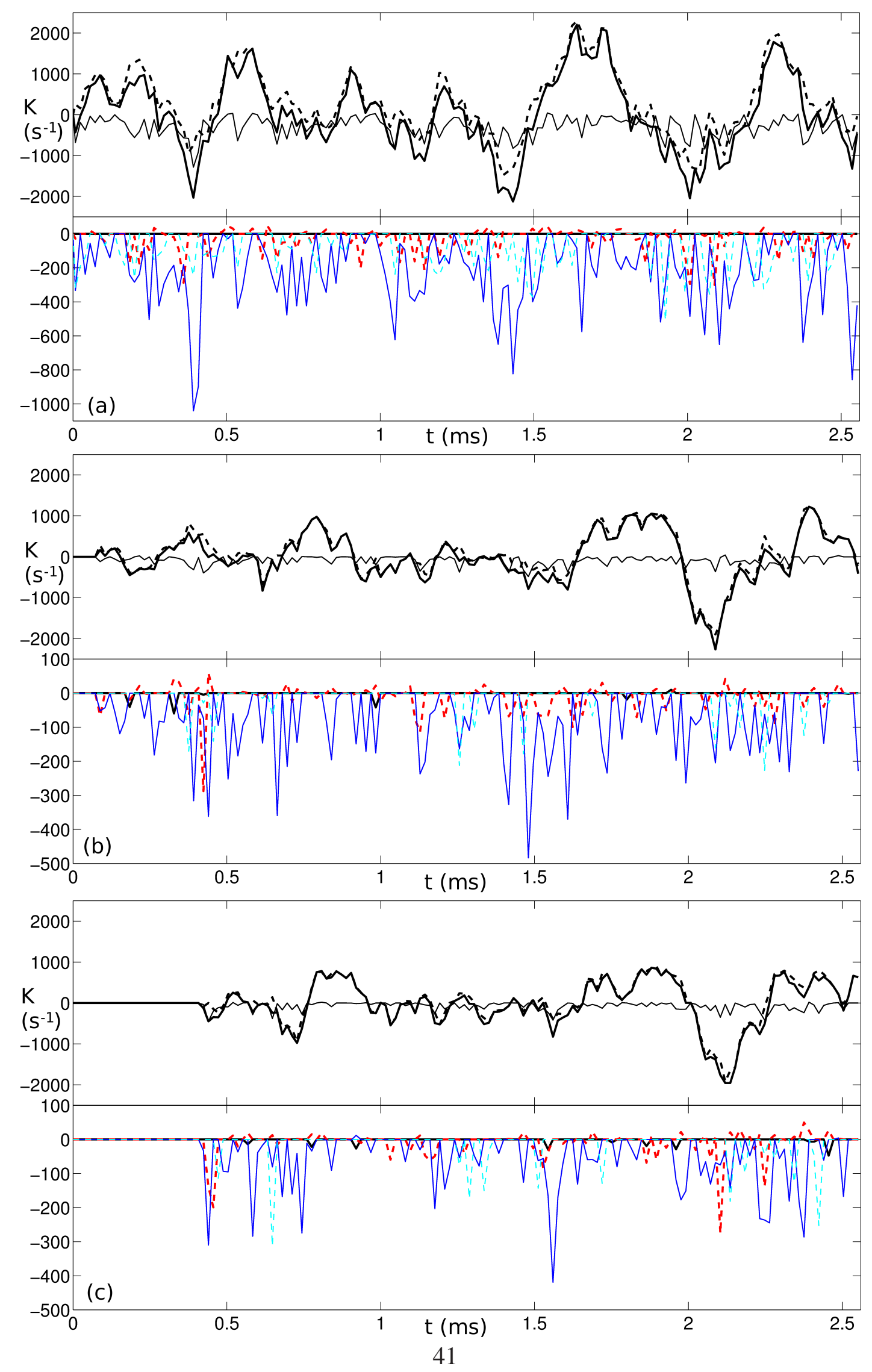


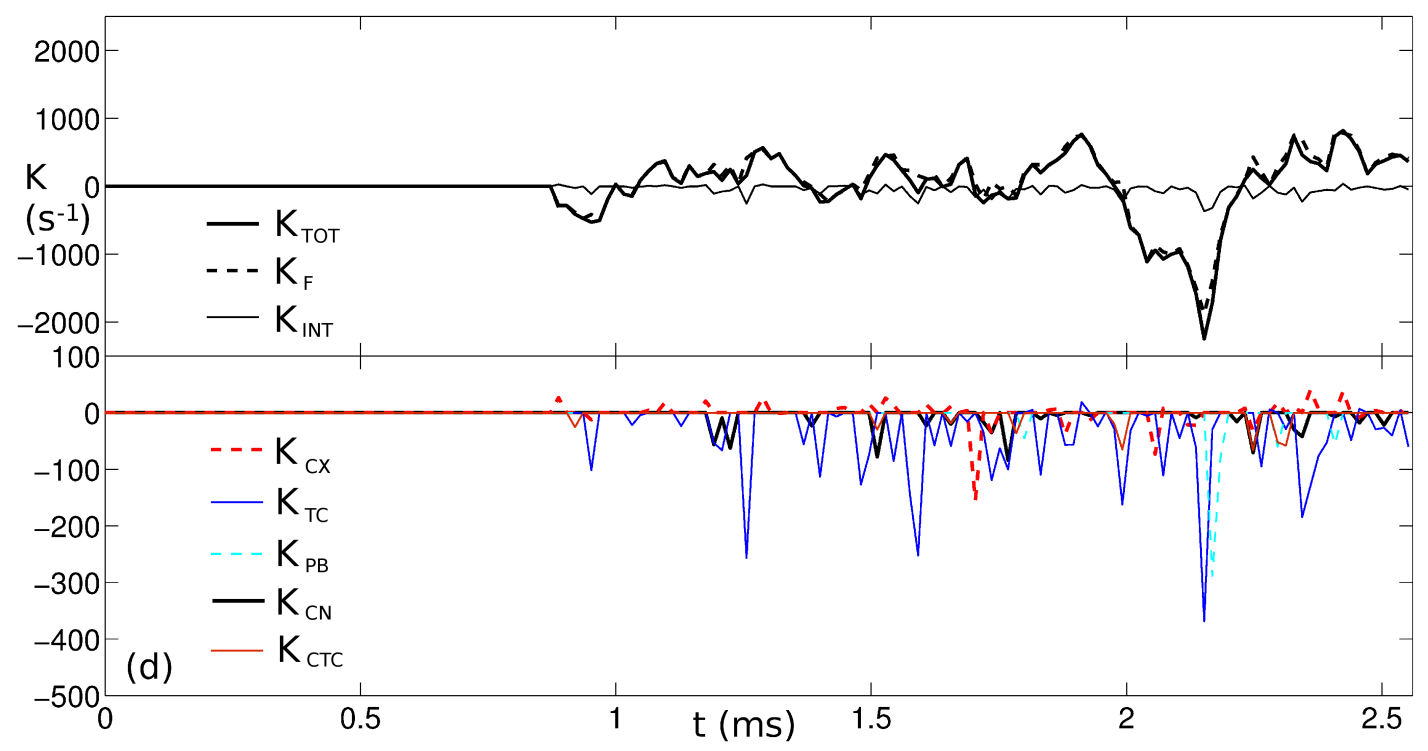

Figure 11: Stretch rates of the $c=0.8$ isosurface for cases flames A-D ((a)-(d) respectively). Top: total stretch rate, $K_{T O T}$, flamelet component, $K_{F}$, and interactions component, $K_{I N T}$. Bottom: interaction stretch rates by type. The $x$-axes of figures a-d have been aligned so that conditions at the inlet are identical for each simulation at a given value of $t$. 